\title{
Hybrid GSA-ANN Methods to Forecast Sheath Current of High Voltage Underground Cable Lines
}

\author{
Bahadır Akbal \\ Selçuk University, Engineering Faculty, Department of Electrical and Electronics Engineering Konya, Turkey. \\ Corresponding author. Tel: +903322232034; email: bakbal@selcuk.edu.tr \\ Manuscript submitted April 7, 2017; accepted July 11, 2017. \\ doi: $10.17706 /$ jcp.13.4.417-425
}

\begin{abstract}
Electrical safety is major issue for electric networks, so high voltage underground cable lines have been used instead of overhead line recently in city center and neighborhood. However, sheath current generates on metallic sheath of high voltage underground cable, and sheath current causes major cable faults and electroshock. Single point bonding, solid bonding and cross bonding are used to reduce sheath current and voltage. If sheath current is determined before high voltage underground cable line is installed, the most suitable method can be used to reduce sheath current and voltage. Hence, cable faults and electroshock can be prevented. There are many factors in formation of sheath current. Thus, formulation of sheath current is very complex and difficult. In this case forecasting methods can be used to determine sheath current, and artificial neural network (ANN) is a powerful method for forecasting studies. In this study, Gravitational Search Algorithm (GSA) and artificial neural network (ANN) is used to reduce training error, and hybrid GSA-ANN method is obtained. It is seen at the end of this study that errors of hybrid GSA-ANN method are less than errors of classic ANN.
\end{abstract}

Key words: Artificial neural network, GSA, sheath current, high voltage underground cable line.

\section{Introduction}

Overhead and high voltage underground cable lines are used for transmission and distribution of electrical energy, and overhead lines are generally used for transmission and distribution of electrical energy because its cost is less than cost of high voltage underground cable lines. However, electrical safety is important issue for electrical networks, and high voltage underground cable line is more safety than overhead lines because high voltage underground cables have an insulation layer. Recently, overhead lines have been converted to high voltage underground cable lines in city center and crowded areas. XLPE insulation is generally used in underground cables, and metallic sheath is used to protect XLPE insulation. High voltage cable is shown in Fig. $1[1]$.

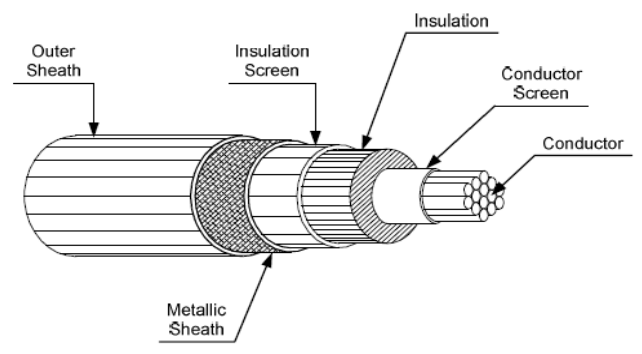

Fig. 1. High voltage underground cable. 
However, sheath current generates on metallic sheath of high voltage underground cable. The sheath current increases cable temperature and the sheath voltage of cable, so cable ampacity reduces, and electro shock risk occurs for human due to high sheath voltage. Also, the sheath current causes major insulation faults. Thus, bonding methods are used to reduce sheath voltage and current. Single point bonding, solid bonding and cross bonding methods are used as bonding methods in IEEE 575-1988 standart. These bonding methods are shown in Fig. 2, Fig. 3 and Fig. 4.

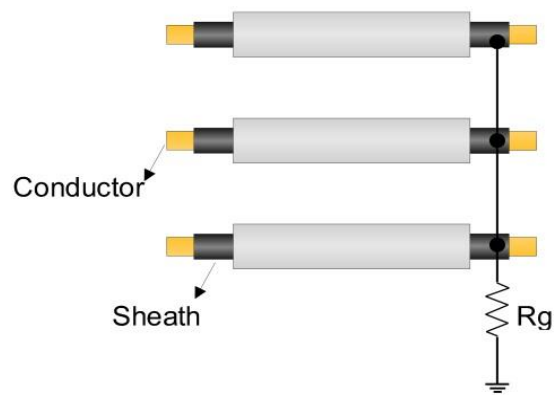

Fig. 2. Single-poind bonding.

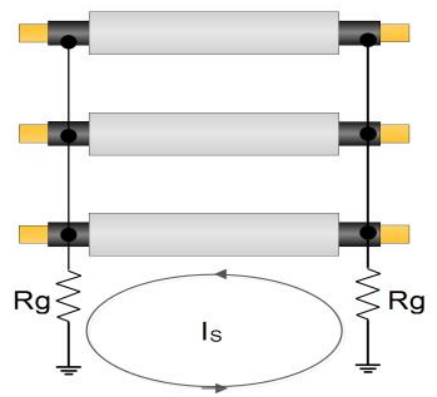

Fig. 3. Solid bonding.

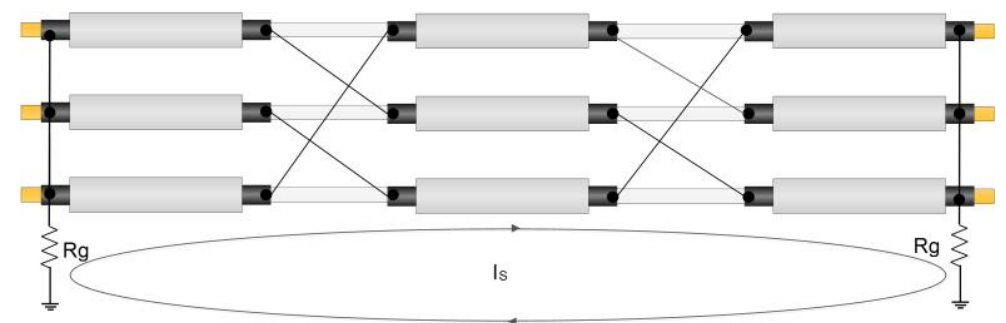

Fig. 4. Cross bonding.

Sheath current does not generate in single point bonding method because sheath circuit is open. However, single point bonding method should not be used in long high voltage underground lines because high voltage occurs on cable sheath due to long length. Solid bonding and cross bonding methods are used for long high voltage underground cable lines. Also, sheath voltage and current can be reduced by using solid bonding and cross bonding method. Sheath current of solid bonding method is less than sheath current of cross bonding method. Also, sheath current of cross bonding is less than sheath current of solid bonding [2]-[6]. Namely, these methods can be used according to problem. If the sheath current is important problem, cross bonding can be used. If the sheath voltage is important problem, solid bonding can be used. However, primarily the sheath current must be determined to solve problems.

If sheath current of high voltage underground cable line is determined before this line is installed, the most 
suitable method can be selected to reduce sheath current and voltage. Thus, cable faults and electroshock risks are prevented; also cable performance can be increased. The sheath current of high voltage underground cable line can be determined by using forecasting method before this line is installed. Artificial neural network (ANN) is powerful forecasting method, and it is used different studies in electrical engineering [7]-[9]. If sheath current data can be obtained, artificial neural network is trained by using these data, so sheath current of new high voltage underground cable line is determined by ANN. Primarily, formation conditions of sheath current should be examined, so sheath current data can be obtained to train ANN.

Unbalanced phase is major factor in formation of the sheath current. If unbalanced phase current amount between phases increases, the sheath current increases [10]. Cable configuration of phases is another factor in formation of the sheath current [11]. Cable configurations are trefoil and flat formations [12]. These configurations are shown in Fig. 5.

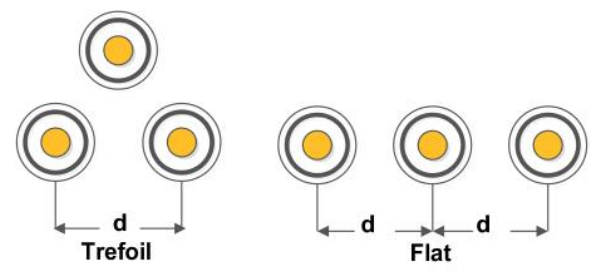

Fig. 5. Cable configurations.

Magnetic flux density is effect to formation steath current [13]. It is seen that there are many factors in formation sheath current. Thus, formulation of the sheath current is very complex and difficult process. Namely, usage of ANN can be advantage for this study. In ANN, training error is major issue, and it should be made minimum value. In literature, hybrid ANN methods are used for minimization of training error of ANN. In this study, Gravitational Search Algorithm (GSA) is used for hybrid ANN. The sheath current data for training of hybrid ANN are generated in PSCAD/EMTDC simulation program. Hence, sheath current of high voltage underground cable line is determined before this line is installed, so the most suitable bonding method can be selected to prevent cable faults and electroshock risks.

\section{Material and Method}

Electromagnetic transient programs are generally used in high voltage underground cable studies [14]. In this study, primarily high voltage cable is modeled in PSCAD/EMTDC simulation program. The modeled cable is shown in Fig. 6.

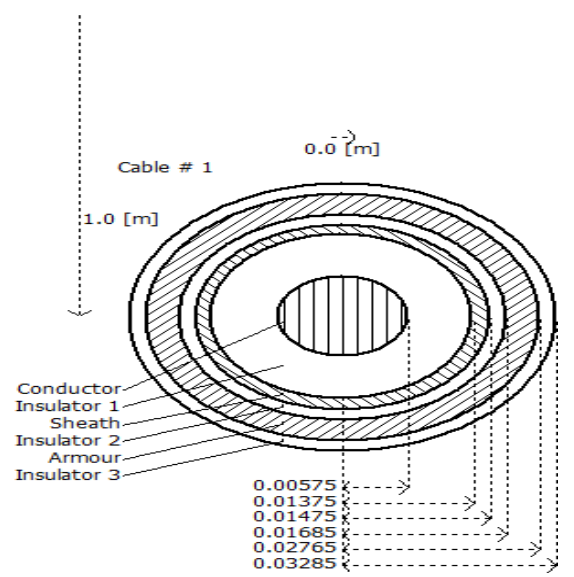

Fig. 6. The modeled high voltage underground cable. 
It is seen at the end of the simulation studies that cable length (L), distance of between phases (d), unbalanced phase current (IUB) and grounding resistance (Rg) are effective in formation of the steath current of the high voltage underground cable lines. These factors are used to generate different high voltage cable lines, and simulations of these cable lines are made in PSCAD/EMTDC to obtain sheath currents of these lines. Thus, the real values of sheath current of different lines are used for training process of ANN.

Working principle of ANN is based on mathematical model of human learning, and the basic element of ANN is neuron. Also ANN occurs from input layer, hidden layer and output layer. The basic schema of ANN is shown in Fig. 7.

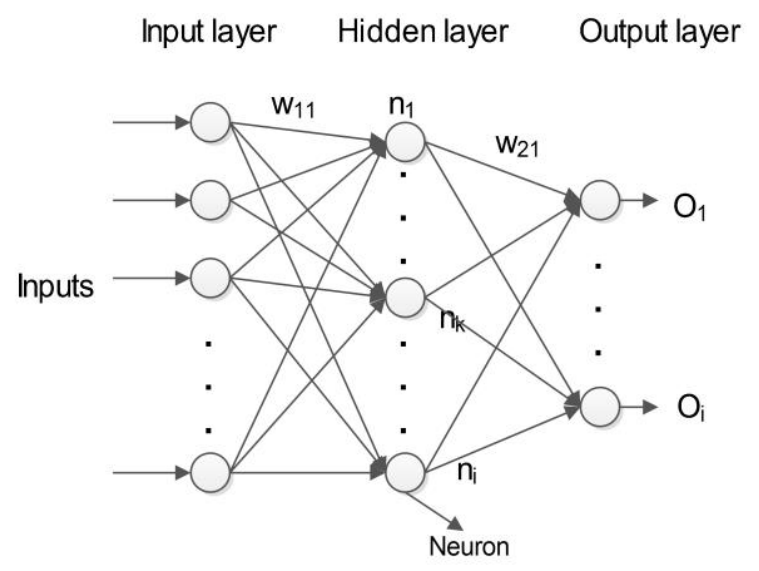

Fig. 7. The basic schema of ANN.

Neurons work as a transfer function. This transfer function is shown in Equation (1), and a neuron working schema is shown in Fig. 8.

$$
y_{i}=f_{i}\left(\sum_{j=1}^{n} w_{i j} \times x_{j}+b_{i}\right)
$$

where, $x_{j}$ is input, $w_{i j}$ is weight, bi is bias, $f_{i}$ is transfer function, and $y_{i}$ is output of the neuron.

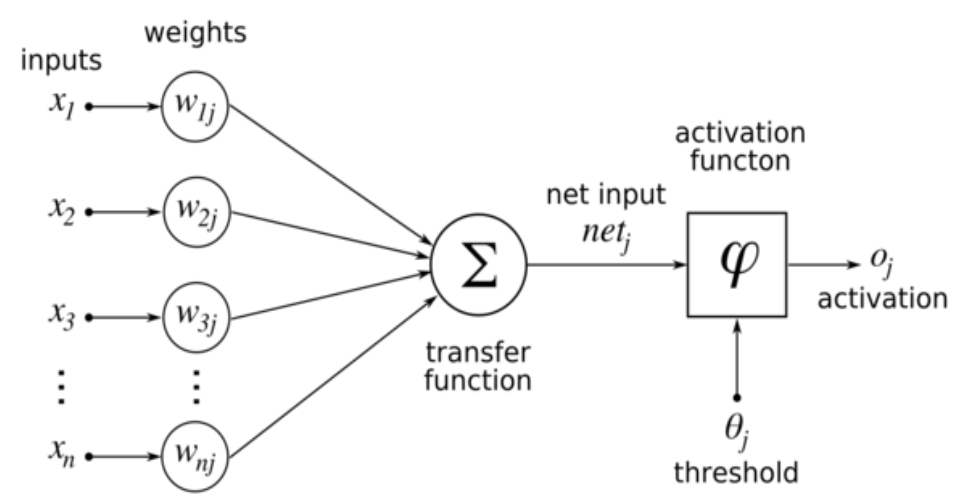

Fig. 8. Neuron working schema.

Activation functions can be sorted as step function, linear function, log-sigmoid function, tan-sigmoid function, softmax function, and gaussian function. Primarily, training process is implemented in ANN, and training error is calculated. Mean square error (MSE) method is used in calculation of training error. MSE is shown in Equation (2). 


$$
E(t)=\frac{1}{n} \sum_{i=1}^{n}(p(i)-o(i))^{2}
$$

where, $E(t)$ is estimation error at tth iteration, $p(i)$ is the desired value for ith output, $o(i)$ is the actual value for ith output.

Weights of ANN should be updated to reduce training error, so Equation (3) is used to update weights in classic ANN.

$$
w_{i}(t+1)=w_{i}(t)+\Delta w_{i}(t)
$$

In hybrid ANN, Equation (3) is not used to update weights, and optimization methods are used instead of Equation (3) to update weights of ANN. Hybrid ANN working schema is shown in Fig. 9, and genetic algorithm is used as optimization methods in this study.

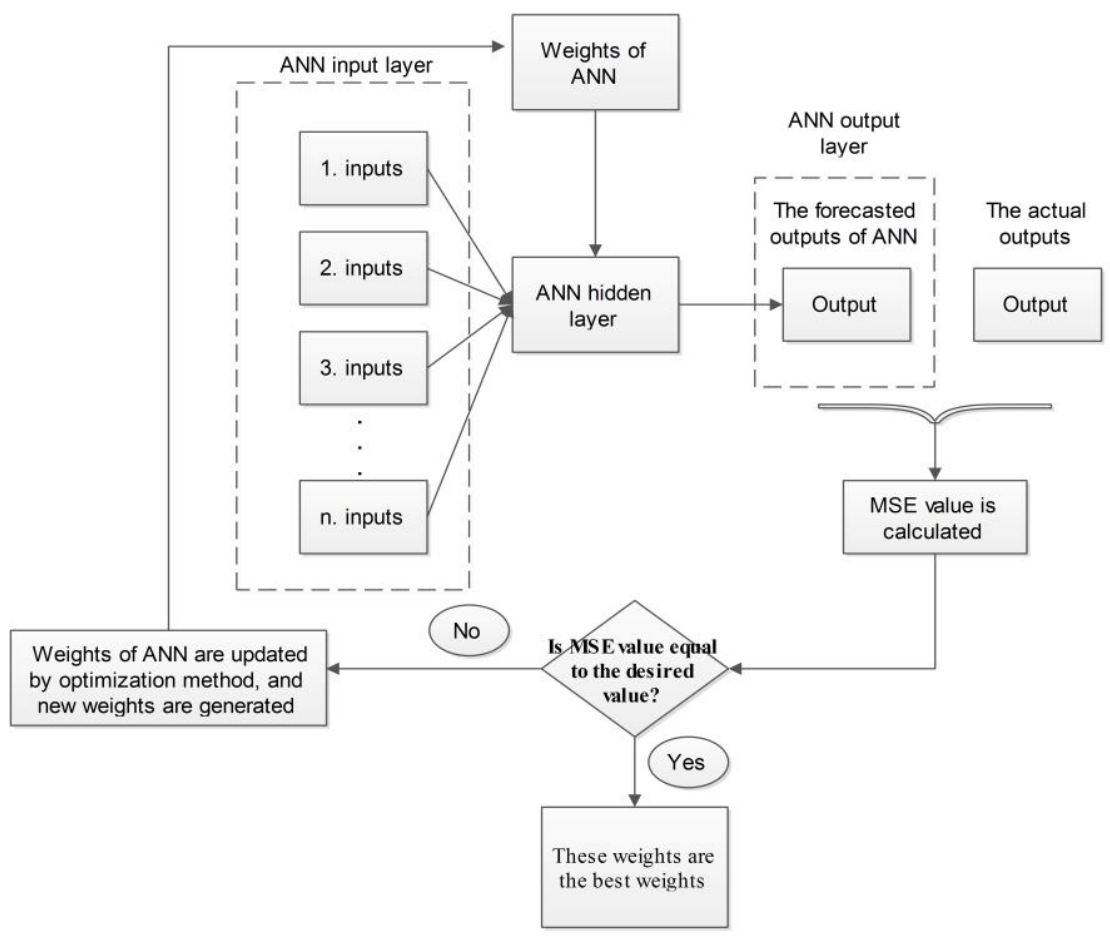

Fig. 9. Hybrid ANN working schema.

\subsection{Gravitational Search Algorithm}

GSA is based on Gravitational law which was found by Newton. Each particle which is defined in Gravitational law is defined as agent in GSA. According to gravitational law, the other particles are attracted by gravitational force of each particle. Gravitational force is affected by particles masses. The particle which has heavier mass attracts the other particles, and the other particles move towards the particle. In GSA, if agent has heavier mass, fitness value of agent is higher value, and it is near to optimum solution. Each agent has four features. These feature are position $\left(X_{i i}\right)$, inertial mass $\left(M_{i i}\right)$, active gravitational mass $\left(M_{a i}\right)$ and passive gravitational mass $\left(M_{p i}\right) . M_{a i}=M_{p i}=M_{i i}$ is calculated with Eq. (4) [15].

$$
m_{i}(t)=\frac{f i t(t)-\operatorname{worst}(t)}{\operatorname{best}(t)-\operatorname{worst}(t)}
$$




$$
M_{i}(t)=\frac{m_{i}(t)}{\sum_{j=1}^{N} m_{j}(t)} \quad i=1,2, \ldots . N
$$

If agent number is $N$ in search space, each agent position can be defined by Eq. (6).

$$
X_{i}=\left(x_{i}^{1} \ldots x_{i}^{d} \ldots x_{i}^{n}\right) \quad i=1,2, \ldots . N
$$

$n$ is agent dimension, and agents occur particles. Namely, is defined as variable of agent. $F$ is gravitational force between ith agent and jth agent at the $t$ time. Gravitational force is shown in Eq. (7).

$$
x_{i}^{d}(t+1)=x_{i}^{d}(t)+v_{i}^{d}(t+1)
$$

$M_{i}$ is mass of ith agent, $M_{j}$ is mass of jth agent, $R_{i j}(t)$ is the Euclidian distance between ith agent and jth agent, and it is defined Eq. (8). $G(t)$ is gravitational constant and is defined Eq. (9).

$$
\begin{aligned}
& R_{i j}(t)=\left\|X_{i}(t), X_{j}(t)\right\|^{2} \\
& G(t)=G_{0} \cdot e^{-\beta\left(t / t_{\max }\right)}
\end{aligned}
$$

$G_{0}$ is an initial value, $\beta$ is constant value, $t$ is the current iteration number, $t_{\max }$ is maximum iteration number, and total force on ith agent is defined with Eq. (10) at $t$ time.

$$
F_{i}^{d}(t)=\sum_{j \in K_{\text {best }}, j \neq i}^{N} \operatorname{rand}_{j} \cdot F_{i j}^{d}(t)
$$

rand $_{j}$ is random number between 0 and $1, K_{\text {best }}$ is the first agents which have the best fitness value. Acceleration of agent is calculated with Eq. (11). Velocity and position are updated with Eq. (12) and Eq. (13) according to acceleration of agent at the $t$ time.

$$
\begin{gathered}
a_{i}^{d}(t)=\frac{F_{i}^{d}(t)}{M_{i i}(t)} \\
v_{i}^{d}(t+1)=\operatorname{rand}_{i} \cdot v_{i}^{d}(t)+a_{i}^{d}(t) \\
x_{i}^{d}(t+1)=x_{i}^{d}(t)+v_{i}^{d}(t+1)
\end{gathered}
$$

$x_{i}^{d}(t)$ is position of ith agent, $v_{i}^{d}(t)$ is velocity of agent, and $\operatorname{rand}_{j}$ is random number between 0 and 1 at the $t$ time. Working principle of GSA as follows [14]:

- Initial population is occurred, and positions of agents are determined as randomly.

- Fitness values of agents are calculated.

- Best and worst agents are selected in population, and $G$ is updated.

- $M$ and a of agents are calculated, and velocity and position of agents are updated.

After these steps are implemented, fitness values of the updated agents are calculated. If optimum value 
is found, or the maximum iteration number is reached, program is stopped. Otherwise, the steps which are between 2 and 4 are continuously implemented.

\section{Experimental Results}

Table 1. Simulation Results of Different Cable Lines

\begin{tabular}{|l|l|l|l|l|l|}
\hline Line & IUB & $\mathrm{L}$ & $\mathrm{d}$ & $\mathrm{R}_{\mathrm{g}}$ & $\mathrm{I}_{\mathrm{SA}}$ \\
\hline 1 & 464.120 & 700 & 0.1 & 40 & 3.3293 \\
\hline 2 & 464.120 & 700 & 0.1 & 50 & 2.6640 \\
\hline 3 & 464.120 & 500 & 0.5 & 25 & 3.5544 \\
\hline 4 & 464.120 & 500 & 0.5 & 30 & 2.9630 \\
\hline 5 & 464.120 & 500 & 0.5 & 35 & 2.5402 \\
\hline 6 & 464.120 & 500 & 0.5 & 40 & 1.9762 \\
\hline 7 & 464.120 & 500 & 0.5 & 50 & 1.7787 \\
\hline 8 & 464.120 & 1000 & 0.5 & 40 & 4.3609 \\
\hline 9 & 464.120 & 1000 & 0.5 & 50 & 3.4897 \\
\hline 10 & 224.630 & 1000 & 0.1 & 30 & 2.8493 \\
\hline 11 & 224.630 & 1000 & 0.1 & 40 & 2.1388 \\
\hline 12 & 224.630 & 1000 & 0.1 & 50 & 1.7116 \\
\hline
\end{tabular}

Table 2. Forecasting Results of ANN and GSA-ANN

\begin{tabular}{|c|c|c|c|}
\hline \multirow{2}{*}{ Line } & \multirow{2}{*}{ ISA $_{\text {SA }}$} & \multicolumn{2}{|c|}{ ISF $(\mathrm{A})$} \\
\cline { 3 - 4 } & & ANN & GSA-ANN \\
\hline 1 & 3.3293 & 4.5406 & 3.0124 \\
\hline 2 & 2.6640 & 4.5406 & 3.0696 \\
\hline 3 & 3.5544 & 4.5407 & 2.6127 \\
\hline 4 & 2.9630 & 4.5406 & 2.6413 \\
\hline 5 & 2.5402 & 4.5406 & 2.6698 \\
\hline 6 & 1.9762 & 4.5406 & 2.6984 \\
\hline 7 & 1.7787 & 4.5406 & 2.7556 \\
\hline 8 & 4.3609 & 4.5407 & 3.4825 \\
\hline 9 & 3.4897 & 4.5407 & 3.5396 \\
\hline 10 & 2.8493 & 4.5413 & 3.7457 \\
\hline 11 & 2.1388 & 4.5407 & 3.8029 \\
\hline 12 & 1.7116 & 4.5407 & 3.8601 \\
\hline
\end{tabular}

In this study, hybrid GSA-ANN method is used to determine the sheath currents for new high voltage underground cable lines. Iteration number is 500, and population size is 10 for GSA. Primarily, training process is made for hybrid GSA-ANN and classic ANN. In training process, different 59 high voltage underground cable line are generated by PSCAD/EMTDC, and sheath current of these lines are measured in PSCAD/EMTDC. Hence, 59x5 data matrix is generated for training process. $\mathrm{I}_{\mathrm{UB}}, \mathrm{L}, \mathrm{d}$ and Rg are used as input parameter. The forecasted sheath current is used output parameter, and training error is calculated by using actual sheath current and the forecasted sheath current. Training error of hybrid GSA-ANN is 0.2381 , and training error of classic ANN is 1.8166. Feed forward backpropagation neural network architecture is used in classic ANN. The best weights which make minimum MSE value are obtained at the end of training process, and the best weights are used to forecast sheath current in forecasting process. In this study, 
forecasting data which are shown in Table 1 are used to forecast sheath current. In Table 1, cable length (L), distance of between phases (d), unbalanced phase current $\left(\mathrm{I}_{\mathrm{UB}}\right)$ and grounding resistance $(\mathrm{Rg})$ are shown. Namely, different 12 high voltage cable lines are designed, and real sheath current values of the designed high voltage underground cable lines are measured in PSCAD/EMTDC. Also, real values of sheath currents (ISA) are shown in Table 1.

Forecasting error of hybrid GSA-ANN is 0.2852 , and forecasting error of classic ANN is 1.7610. The forecasted sheath currents ( $\mathrm{I}_{\mathrm{SF}}$ ) of hybrid GSA-ANN and classic ANN are shown in Table 2. Also ISA is shown in Table 2. It is seen that training and forecasting errors of hybrid GSA-ANN are less than training and forecasting errors of classic ANN.

\section{Conclusion}

The sheath current causes major insulation faults and electroshock risks in high voltage underground lines. Single point bonding, solid bonding and cross bonding are used to reduce the sheath current and voltage. The sheath current should be determined to select the most suitable method. Also if sheath current can be determined before high voltage underground cable line is installed, the most suitable method is selected to reduce sheath current and voltage, so cable faults and electroshock risks can be prevented early. ANN can be used to forecast the sheath current of high voltage underground cable line. However, iteration number and training data should be increased to reduce training and forecasting errors of classic ANN, so computational time increases. In this study, hybrid GSA-ANN method is used to forecast steath current of high voltage underground cable line which will be installed as a new line. It is seen that computational time, training error and forecasting error of hybrid GSA-ANN is less than training and forecasting errors of classic ANN.

\section{Acknowledgment}

This study was supported by 16701773 number Selçuk University Coordinatorship of Scientific Research Projects.

\section{References}

[1] Tziouvaras, A. D. (2006). Protection of high-voltage AC cables. Proceedings of IEEE Power Systems Conference: Advanced Metering, Protection, Control, Communication, and Distributed Resources (pp. 316-328 ). South Carolina: Clemson.

[2] Robert, B. (2009). Multiconductor analysis of underground power transmission systems: EHV AC cables. Electr Pow Syst Res, 79, 27-38.

[3] Riba, R., Antoni, G., \& Alabern, M. (2007). Circulating sheath currents in flat formation underground power lines. RE\&PQJ, 1, 61-65.

[4] Li, Z., Du B. X., Wang, L., Yang, C., \& Liu, H. J. (2012). The calculation of circulating current for the single-core cables in smart grid. Proceedings of IEEE Innovative Smart Grid Technologies - Asia (pp. 1-4).

[5] Stanislaw, C., Krzysztof, D., Jacek, K., \& Zbigniew, L. (2014). Calculation of induced sheath voltages in power cables - single circuit system versus double circuit system. J Inf Contr \& Manage Syst., 12, 113-123.

[6] Jung, C. K., Lee, J. B., \& Kang, J. W. (2007). Sheath circulating current analysis of a cross-bonded power cable systems. J Electr Eng \&Technol, 2, 320-328.

[7] Wictor, C., \& Mo-Shing, C. (2000). Very short-term load forecasting using artificial neural networks. IEEE Transactions on Power Systems, 15, 263-268.

[8] Renuka, A. (2012). Long term electric load forecasting using neural networks and support vector 
machines. International Journal of Computer Science and Technology, 3, 266-269.

[9] Weigerta, T., Tianb, Q., \& Lianb, Q. (2011). State-of-charge prediction of batteries and battery-supercapacitor hybrids using artificial neural networks. Journal of Power Sources, 196, 4061-4066.

[10] Jung, C. K., Lee, J. B., \& Kang, J. W. (2007). Sheath circulating current analysis of a cross-bonded power cable systems.J Electr Eng \&Technol, 2, 320-328.

[11] Gouda, O. E., \& Farag, A. (2012). Factors affecting the sheath losses in single-core underground power cables with two-points bonding method. Int J Electr \& Comp Eng, 2, 7-16.

[12] Riba, R., \& Albern, M. (2006). Effects of the circulating sheath currents in the magnetic field generated by an underground power line. Proceedings of International Conference on Renewable Energies and Power Quality (pp. 1-5). Palma: Mallorca.

[13] Lai, G., Feng, Y., Huang, H. M., \& Ching-Tzong, S. (2008). Optimal connection of power transmission lines with underground power cables to minimize magnetic flux density using genetic algorithms. IEEE T Power Deliver, 23, 1553-1560.

[14] Stella, G. U., Bjørn, G., Leth, B. C., \& Wojciech, W. (2011). Field test and simulation of a 400-kV cross-bonded cable system. IEEE T Power Deliver, 26, 1403-1410.

[15] Rashedi, E., Nezamabadi, H., \& Saryazdi, S. (2009). GSA: A gravitational search algorithm. Elsevier Information Sciences, 179, 2232-2248.

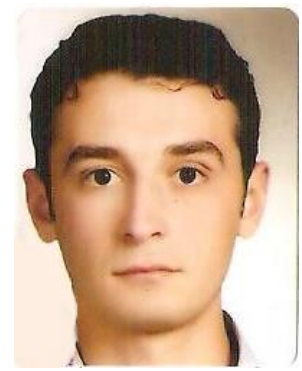

B. Akbal was born in Elbistan, Turkey, in 1983. He received the B.Sc. degree in Electrical-Electronics Engineering Department from Inonu University, Malatya, Turkey, in 2006 and the M.Sc. degree in Electrical-Electronics Engineering Department from Selçuk University, Konya, Turkey in 2011 and then received the Ph.D degree in Electrical-Electronics Engineering Department from Selçuk University, Konya, Turkey in 2015. He has studied in Electrical-Electronics Engineering Department from Selçuk University as an assistant professor. His areas of research include high voltage engineering, electrical energy transmission and distribution, power system stability, optimization techniques and artificial intelligent, industrial automation, power quality. 